\title{
Effect of dietary supplementation with increasing doses of docosahexaenoic acid on neutrophil lipid composition and leukotriene production in human healthy volunteers
}

\author{
Françoise Stanke-Labesque ${ }^{1,2,3 *}$, Patrick Molière ${ }^{1}$, Jeanine Bessard ${ }^{2}$, Martine Laville ${ }^{1}$, Evelyne Véricel ${ }^{1}$ \\ and Michel Lagarde ${ }^{1}$ \\ ${ }^{1}$ Université de Lyon; INSERM UMR 870; INSA-Lyon, RMND, F-69621; INRA UMR 1235; Université Lyon 1; Hospices Civils \\ de Lyon, France \\ ${ }^{2}$ Laboratory of Pharmacology, Grenoble University Hospital, INSERM, ERI17, Grenoble F-38042, France \\ ${ }^{3}$ Faculté de Médecine, Université Grenoble 1, IFR1, Grenoble F-38042, France \\ (Received 12 September 2007 - Revised 19 December 2007 - Accepted 8 January 2008 - First published online 28 February 2008)
}

$n$-3 PUFA supplementation helps in the prevention or treatment of inflammatory diseases and CVD. However, many supplementations reported so far are either a combination of $n-3$ PUFA or used large daily amounts of $n-3$ PUFA dosages. The present study investigated the influence of increasing dose intake of DHA on the fatty acid composition of phospholipids in neutrophils and on their capability to produce leukotrienes (LT) $\mathrm{B}_{4}$ and $\mathrm{B}_{5}$ in vitro. Twelve healthy volunteers were supplemented with increasing daily doses of DHA (200, 400, 800 and $1600 \mathrm{mg}$, each dose in TAG containing DHA as the only PUFA and for a 2-week period). At the end of each supplementation period, neutrophil fatty acid composition, and $\mathrm{LTB}_{4}$ and $\mathrm{LTB}_{5}$ production were determined by GC and liquid chromatography-tandem MS, respectively. The DHA/arachidonic acid ratio increased in a dose-dependent manner with respect to the increasing doses of DHA supplementation and was significantly different from baseline after supplementation with either 400,800 or $1600 \mathrm{mg}$ DHA. The $\mathrm{LTB}_{5} / \mathrm{LTB}_{4}$ ratio was significantly increased compared to baseline after supplementation with 800 and $1600 \mathrm{mg}$ DHA. $\mathrm{LTB}_{5} / \mathrm{LTB}_{4}$ and DHA/arachidonic acid ratios were correlated $(r 0.531, P<0 \cdot 0001)$. The present data suggest that both changes in neutrophil lipid composition and LT production occurred with daily supplementation with 800 and $1600 \mathrm{mg}$ DHA. The clinical benefits associated with these doses of DHA in inflammatory diseases remain to be investigated.

Dietary DHA: Leukotrienes: Polymorphonuclear cells

In the past century, dietary and other environmental changes have been considered to be among the major causes of the rapid expansion of chronic diseases. In western countries, the increased incidence of inflammatory diseases has partly been attributed to the abundant consumption of $n$ - 6 PUFA with lower ingestion of $n-3$ PUFA. In this context, nutritional manipulations help in the prevention and/or treatment of various inflammatory diseases ${ }^{(1)}$.

Linoleic and $\alpha$-linolenic acids are essential fatty acids and must therefore be supplied in the diet. They are the precursors of the $n-6$ and $n-3$ series of fatty acids, respectively.

Linoleic acid may be converted to arachidonic acid (AA), which is subsequently incorporated into cell membrane phospholipids. Further metabolism of released AA by 5-lipoxygenase yields the four-series leukotrienes (LT; e.g. $\mathrm{LTB}_{4}$ ), which possess potent inflammatory properties. Conversely, $\alpha$-linolenic acid may be converted into EPA and DHA, which are also subsequently incorporated into cell membrane phospholipids.
It may be stressed, however, that the conversion of linolenic acid into long-chain derivatives is weak in men ${ }^{(2)}$, which means that EPA and especially DHA must be consumed as preformed acids, i.e. from marine food. EPA yields the fiveseries eicosanoids, including $\mathrm{LTB}_{5}$, through the 5-lipoxygenase pathway. Interestingly, many of the eicosanoids produced from EPA possess markedly reduced inflammatory properties compared with the AA-derived lipid mediators and may even exert antagonistic functions (for a review, see Calder ${ }^{(3)}$ ).

Dietary supplementations reported so far are a combination of DHA/EPA ${ }^{(4-9)}$; consequently, there is a lack of clarity regarding the differential biological effects of EPA and DHA. In addition, and although the clinical benefits of $n-3$ PUFA in CVD are observed with sub-gram doses ${ }^{(10)}$, the underlying cellular changes induced by DHA or EPA supplementation have been investigated using daily amounts of $n$-3 PUFA greater than $1 \mathrm{~g} / \mathrm{d}^{(4-9,11)}$. Few studies have assessed the effects of supplementation with lower doses of DHA and

Abbreviations: AA, arachidonic acid; LT, leukotriene.

* Corresponding author: Dr Françoise Stanke-Labesque, Laboratory of Pharmacology, Grenoble University Hospital, BP 217, F-38043 Grenoble Cedex 9, France; HP2 Laboratory, INSERM EA 3745 ERI 0017, Joseph Fourier University, University of Medicine, BP 170, F-38042 Grenoble Cedex 9 , France, fax + 33476768938 , email FStanke@chu-grenoble.fr 
the data reported are conflicting. In platelets ${ }^{(12)}$ or lymphocytes $^{(13)}$ from elderly people, the incorporation of DHA in lipid membranes occurs after daily supplementation with $150 \mathrm{mg}$ DHA + $30 \mathrm{mg}$ EPA. In contrast, Di Stasi et al. ${ }^{(8)}$ found that 12-week oral supplementation with $1 \mathrm{~g} n$-3 PUFA failed to change DHA content in platelet and mononuclear cell membranes. To our knowledge, the influence of low dosages of $n$-3 PUFA on the lipid content of neutrophils and their capability to synthesize $\mathrm{LTB}_{4}$ and $\mathrm{LTB}_{5}$ remains to be determined. The present study investigated the dose-dependent incorporation of DHA in neutrophils from healthy volunteers and the subsequent ex vivo production of $\mathrm{LTB}_{4}$ and $\mathrm{LTB}_{5}$.

\section{Materials and methods}

\section{Clinical study}

The protocol was approved by the local ethic committee in accordance with the Declaration of Helsinki, and all the participants gave written informed consent.

Twelve healthy males (mean age 58 years; age range 53-65 years) were included. Volunteers were excluded if they were taking any anti-inflammatory medication, and had been diagnosed as having CVD, diabetes, liver or endocrine dysfunction, or cancer. All participants supplemented their usual diet with daily consumption of capsules of Pro-Mind (Decoma, Belgium). Each capsule contained $200 \mathrm{mg}$ DHA in TAG from algal oil, $0.125 \mathrm{mg}$ DL- $\alpha$-tocopherol and $0.125 \mathrm{mg}$ ascorbic palmitate. The supplementation consisted of the daily ingestion of successively 200, 400, 800 and $1600 \mathrm{mg}$ DHA for 2 weeks for each dose, without interruption. The volunteers were instructed to keep their usual diet regimens throughout the study.

Blood samples were collected after overnight fasting 2 weeks before DHA supplementation started (DHA $0 \mathrm{mg}$ ), and at the end of each DHA dose supplementation period (200, 400, 800 and $1600 \mathrm{mg}$ ). Blood samples were also collected 5 weeks after supplementation was arrested.

\section{Isolation of human polymorphonuclear cells}

Venous blood was collected on citrate as anticoagulant. Polymorphonuclear cells were isolated by dextran sedimentation, followed by Ficoll-Paque centrifugation as previously described $^{(14)}$. Cellular viability was greater than $98 \%$ as judged by the Trypan blue exclusion method.

Polymorphonuclear cells $\left(2 \times 10^{6}\right.$ cells $\left./ \mathrm{ml}\right)$ were suspended in $450 \mu$ l Tyrode-HEPES buffer $(\mathrm{pH} 7 \cdot 4)$ containing $0 \cdot 133 \mathrm{~g} / \mathrm{l}$ $\mathrm{CaCl}_{2}$ and $0 \cdot 1 \mathrm{~g} / 1 \mathrm{MgCl}_{2}$ and incubated for $5 \mathrm{~min}$ at $37^{\circ} \mathrm{C}$ with the calcium ionophore A23187 at $0.5 \mu \mathrm{mol} / \mathrm{l}$, or vehicle (ethanol $0.05 \%$ ). The reaction was stopped by the addition of $500 \mu \mathrm{l}$ cold methanol-acetonitrile $(\mathrm{v} / \mathrm{v})$. The supernatants were stored at $-80^{\circ} \mathrm{C}$ for later LT quantification as well as the cell residues for fatty acid composition.

\section{Analysis of total lipids in neutrophils}

Determination of mole quantities of total lipids was accomplished by GC as previously described ${ }^{(15)}$.

\section{Leukotrienes $B_{4}$ and $B_{5}$ quantification}

Quantifications of $\mathrm{LTB}_{4}$ and $\mathrm{LTB}_{5}$ were performed on $800 \mu \mathrm{l}$ centrifuged supernatant by liquid chromatography-tandem MS. $\mathrm{LTB}_{4}-\mathrm{d}_{4}(2 \mathrm{ng})$ was added on each sample as an internal standard. Solid-phase extraction was performed by using C18 (EC) cartridge $(100 \mathrm{mg} / 10 \mathrm{ml})$ purchased from International Sorbent Technology (UK). Methanolic extracts were dried under nitrogen flow at room temperature and reconstituted in $40 \mu \mathrm{l}$ mobile phase: methanol-ammonium formate $(10 \mathrm{mmol} / \mathrm{l} ; 80: 20, \mathrm{v} / \mathrm{v})$. After centrifugation, $10 \mu \mathrm{l}$ were injected into the liquid chromatography-tandem MS system previously described ${ }^{(16)}$. The chromatographic separation was obtained on a Chromasil C8 $5 \mu \mathrm{m}(125 \times 2 \mathrm{~mm})$ column (Macherey-Nagel, France) maintained at $30^{\circ} \mathrm{C}$.

MS acquisitions were made in the negative-ion mode using multiple reaction monitoring with the transitions $\mathrm{m} / \mathrm{z}$ $335.0 \rightarrow 195.1$ for $\mathrm{LTB}_{4}, \mathrm{~m} / z$ 339.1 $\rightarrow 197 \cdot 1$ for $\mathrm{LTB}_{4}-\mathrm{d}_{4}$ and $m / z, 333 \cdot 2 \rightarrow 195 \cdot 1$ for $\mathrm{LTB}_{5}$. The lower limit of quantification was $30 \mathrm{pg} / \mathrm{ml}$ for both LT.

\section{Materials}

Reagents used and their sources were: $\mathrm{A}_{23187}$ and $\mathrm{LTB}_{5}$ from Sigma Aldrich, and $\mathrm{LTB}_{4}$ and $\mathrm{LTB}_{4}-\mathrm{d}_{4}$ from Cayman.

\section{Statistical analysis}

Fatty acid content was calculated as a percentage of total fatty acids in the neutrophil lipids. LT production was expressed as $\mathrm{ng} / 2 \times 10^{6}$ cells. $\mathrm{LTB}_{5} / \mathrm{LTB}_{4}$ ratio was expressed as $\mathrm{LTB}_{5} /$ $\mathrm{LTB}_{4}$ ratio $\times 100$. Data are presented as median (10th to 90th percentiles). Comparisons between baseline and each dose of DHA supplementation were performed using a Friedman test. Subsequent pair-wise comparisons were made with the Wilcoxon test adjusted with the Bonferonni correction for multiple comparisons. Correlations were analysed using the Spearman rank test. $P<0.05$ was considered significant.

\section{Results}

\section{Cell fatty acid composition}

The fatty acid composition of total lipids in neutrophils at baseline and after supplementation with increasing doses of DHA is displayed in Table 1.

The major changes result in a dose-dependent increase of DHA proportions that are significantly different from baseline with the daily supplementation with 400,800 and $1600 \mathrm{mg}$ DHA, respectively. The proportion of 16:0, 18:0, 18:1n-9 and 18:2n-6 remained unchanged (data not shown).

The DHA/AA ratio increased in a dose-dependent manner with respect to the increasing doses of DHA supplementation and was significantly different from baseline for the daily supplementations with 400, 800 and $1600 \mathrm{mg}$ DHA, respectively.

Five weeks discontinuation of DHA supplementation led to a decrease of DHA proportion and DHA/AA ratio, which were similar to those observed at baseline (Table 1).

The proportion of EPA was significantly correlated with the proportion of DHA $(r 0 \cdot 364, P=0 \cdot 0018)$ and was significantly increased compared to baseline after the daily supplementation with $1600 \mathrm{mg}$ DHA (Table 1). 
The proportion of $22: 5 n-3$ was significantly decreased compared to baseline after supplementation with 800 and $1600 \mathrm{mg}$ DHA and remained significantly lower 5 weeks after the arrest of DHA supplementation (Table 1).

\section{Leukotriene production}

As shown in Fig. 1, the $\mathrm{LTB}_{5} / \mathrm{LTB}_{4}$ ratio increased as a function of the doses of DHA and was significantly different from baseline after 800 and $1600 \mathrm{mg}$ DHA.

The $\mathrm{LTB}_{5} / \mathrm{LTB}_{4}$ ratio was correlated with the dose of DHA $(r \quad 0.532, P<0.0001)$. In addition, the $\mathrm{LTB}_{5} / \mathrm{LTB}_{4}$ ratio was significantly correlated with EPA content $(r$ 0.525, $P<0.0001)$, EPA/AA ratio $(r 0.510, P<0.0001)$ and DHA/ AA ratio $(r 0.531, P<0.0001)$.

\section{Discussion}

The present study is the first to demonstrate that dietary supplementation with increasing doses of DHA as the only PUFA induces a dose-dependent incorporation of DHA in neutrophil lipids, and a subsequent dose-dependent increase in the ratio of $\mathrm{LTB}_{5} / \mathrm{LTB}_{4}$ production by neutrophils in response to calcium ionophore challenge. Before DHA supplementation started, the proportion of DHA in total lipids and the production of LT remained stable during a 2-week period (data not shown), attesting both the reliability and the reproducibility of biochemical measurements.

The dietary supplementation with DHA induced major changes in the lipid content from neutrophils. First, the proportion of DHA in neutrophil lipids was significantly increased after 2-week supplementation with $400 \mathrm{mg}$ DHA/d, attesting that incorporation of DHA in lipids from neutrophils could occur after daily supplementation with this low dose of DHA. Conversely, a previous study suggested that $1.8 \mathrm{~g}$ DHA ethyl ester/d was required to significantly increase DHA proportion in neutrophil lipids ${ }^{(8)}$. This discrepancy could be explained by higher bioavailability of DHA-containing glycerides compared to DHA ethyl ester ${ }^{(17)}$. However, with respect to the slow DHA incorporation ${ }^{(18)}$, it could not be excluded that a longer intervention would have been more effective with the lowest dose of DHA. Second, the proportion of EPA significantly increased after the daily intake of $1600 \mathrm{mg}$ DHA, and was significantly correlated to DHA content. Since the dietary supplementation was free of EPA, the data suggest that DHA was likely retroconverted to EPA as previously described ${ }^{(19)}$. Although the design of the present study did not allow determination of which dose of DHA initiates the retroconversion of DHA to EPA, the present data suggest that it might occur only for high doses of DHA. Lastly, and unexpectedly, the proportion of $22: 5 n-3$ (docosapentaenoic acid) was significantly decreased compared to baseline after supplementation with 800 and $1600 \mathrm{mg}$ DHA and remained significantly lowered 5 weeks after the arrest of DHA. With docosapentaenoic acid being an intermediate of EPA and DHA, which both increased in response to highest doses of dietary DHA, it may be speculated that DHA could compete with endogenous docosapentaenoic acid to lower it in lipid stores.

The increased incorporation of DHA in neutrophil lipids was associated with a trend towards an increased $\mathrm{LTB}_{5} / \mathrm{LTB}_{4}$ 


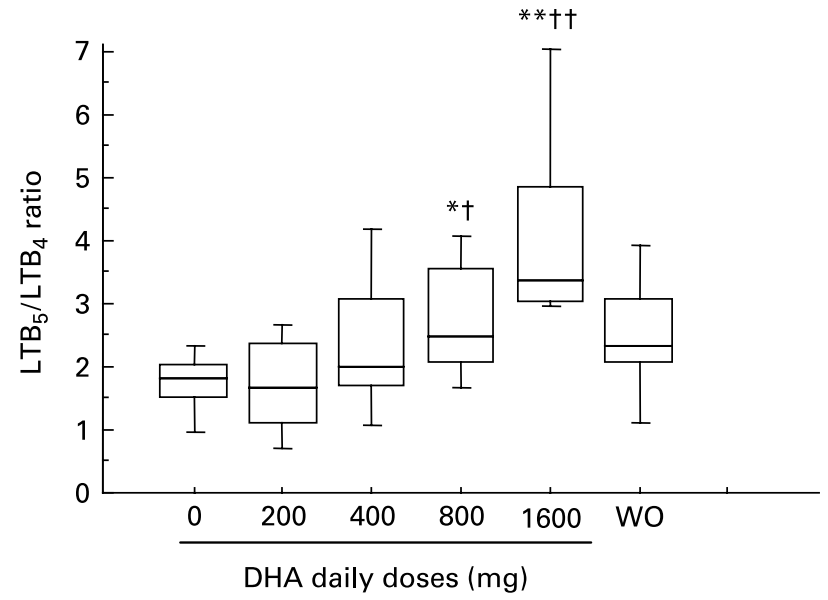

Fig. 1. Changes in leukotriene (LT) production by A23187-stimulated neutrophils from healthy volunteers before and after supplementation with increasing doses of DHA. Data are expressed as $\mathrm{LTB}_{5} / \mathrm{LTB}_{4}$ ratios. Boxes represent values within the interquartile range; whiskers represent the data range; and the lines across the boxes represent median values. WO, washout period. Median values were significantly different from those of the $0 \mathrm{mg}$ DHA dose: ${ }^{\star} P<0.017,{ }^{\star \star} P<0.0079$. Median values were significantly different from those of the washout period: $\uparrow P<0.017, \dagger \dagger P<0.0079$

ratio that reached statistical significance from $800 \mathrm{mg}$ DHA and over. Consistent with the conversion of DHA to EPA as discussed earlier, $\mathrm{LTB}_{5} / \mathrm{LTB}_{4}$ ratio was correlated with DHA/AA and EPA/AA ratios. Since the proportion of AA did not significantly decrease during the DHA supplementation period, the present data suggest that DHA increased $\mathrm{LTB}_{5} / \mathrm{LTB}_{4}$ ratio at least in part through its retroconversion to EPA rather than through an inhibitory effect on AA content.

In the present study, the doses of DHA have not been allocated at random to five separate groups of healthy subjects but have been taken successively by the same subjects. Consequently, it could not be excluded that the increased incorporation of DHA in neutrophil lipids and the subsequent production of LT observed for each dose of DHA may also reflect the effect of cumulative doses of DHA. Results from human studies indeed indicate that the level of DHA reached a plateau after 18 weeks of dietary supplementation ${ }^{(18)}$ and most of the published data reported longer supplementation periods (from 3 weeks to 6 months ${ }^{(5,6,8,9,13)}$ ). However, the present data suggest that changes in total lipid content occurred within 2 weeks and therefore earlier than that reported until now.

In addition, DHA incorporation and ex vivo LT production returned to baseline levels 5 weeks after that the dietary supplementation with the highest dose of DHA was arrested, indicating a high turnover of DHA in cells.

We must acknowledge some limitations in the present study. The sample size and the lack of placebo group imply that the presented data should be considered as preliminary. Further studies are obviously required to confirm them. In addition, although the dose-dependent increase of DHA content provided indirect evaluation of the compliance of subjects, the latter has not been directly measured.

In conclusion, the present data demonstrate that changes in neutrophil lipid composition and $\mathrm{LTB}_{5} / \mathrm{LTB}_{4}$ ratio occurred after daily supplementation with 800 and $1600 \mathrm{mg}$ DHA for
2 weeks. Further studies are needed to investigate the clinical benefits of dietary supplementation with these doses of DHA in inflammatory diseases or CVD.

\section{Acknowledgements}

This work was supported by INSERM and a grant from GLN. F. S.-L. was on temporary assignment in Lyon. There is no conflict of interest and all the authors adhere to the Committee on Publication Ethics guidelines on research and publication ethics.

\section{References}

1. Calder PC (2006) n-3 polyunsaturated fatty acids, inflammation, and inflammatory diseases. Am J Clin Nutr 83, 1505S-1519S.

2. Burdge GC, Finnegan YE, Minihane AM, Williams CM \& Wootton SA (2003) Effect of altered dietary n-3 fatty acid intake upon plasma lipid fatty acid composition, conversion of $\left[{ }^{13} \mathrm{C}\right]$ alpha-linolenic acid to longer-chain fatty acids and partitioning towards beta-oxidation in older men. Br J Nutr 90, 311-321.

3. Calder PC (2002) Dietary modification of inflammation with lipids. Proc Nutr Soc 61, 345-358.

4. Chilton FH, Patel M, Fonteh AN, Hubbard WC \& Triggiani M (1993) Dietary n-3 fatty acid effects on neutrophil lipid composition and mediator production. Influence of duration and dosage. J Clin Invest 91, 115-122.

5. Sperling RI, Benincaso AI, Knoell CT, Larkin JK, Austen KF \& Robinson DR (1993) Dietary omega-3 polyunsaturated fatty acids inhibit phosphoinositide formation and chemotaxis in neutrophils. J Clin Invest 91, 651-660.

6. Kew S, Banerjee T, Minihane AM, Finnegan YE, Muggli R, Albers R, Williams CM \& Calder PC (2003) Lack of effect of foods enriched with plant- or marine-derived n-3 fatty acids on human immune function. Am J Clin Nutr 77, 1287-1295.

7. Mayer K, Fegbeutel C, Hattar K, Sibelius U, Kramer HJ, Heuer KU, Temmesfeld-Wollbruck B, Gokorsch S, Grimminger F \& Seeger W (2003) Omega-3 vs. omega-6 lipid emulsions exert differential influence on neutrophils in septic shock patients: impact on plasma fatty acids and lipid mediator generation. Intensive Care Med 29, 1472-1481.

8. Di Stasi D, Bernasconi R, Marchioli R, Marfisi RM, Rossi G, Tognoni G \& Tacconi MT (2004) Early modifications of fatty acid composition in plasma phospholipids, platelets and mononucleates of healthy volunteers after low doses of n-3 polyunsaturated fatty acids. Eur J Clin Pharmacol 60, 183-190.

9. Gorjao R, Verlengia R, Lima TM, et al. (2006) Effect of docosahexaenoic acid-rich fish oil supplementation on human leukocyte function. Clin Nutr 25, 923-938.

10. Gruppo Italiano per lo Studio della Sopravvivenza nell'Infarto Miocardico (1999) Dietary supplementation with n-3 polyunsaturated fatty acids and vitamin $\mathrm{E}$ after myocardial infarction: results of the GISSI-Prevenzione trial. Lancet 354, 447-455.

11. Kelley DS, Taylor PC, Nelson GJ \& Mackey BE (1998) Dietary docosahexaenoic acid and immunocompetence in young healthy men. Lipids 33, 559-566.

12. Vericel E, Calzada C, Chapuy P \& Lagarde M (1999) The influence of low intake of n-3 fatty acids on platelets in elderly people. Atherosclerosis 147, 187-192.

13. Bechoua S, Dubois M, Vericel E, Chapuy P, Lagarde M \& Prigent AF (2003) Influence of very low dietary intake of 
marine oil on some functional aspects of immune cells in healthy elderly people. Br J Nutr 89, 523-531.

14. Hosni R, Chabannes B, Pacheco Y, Moliere P, Grosclaude M, Perrin Fayolle M \& Lagarde M (1991) Leukotriene $B_{4}$ levels from stimulated neutrophils from healthy and allergic subjects: effect of platelets and exogenous arachidonic acid. Eur J Clin Invest 21, 631-637.

15. Diaz O, Berquand A, Dubois M, Di Agostino S, Sette C, Bourgoin S, Lagarde M, Nemoz G \& Prigent AF (2002) The mechanism of docosahexaenoic acid-induced phospholipase D activation in human lymphocytes involves exclusion of the enzyme from lipid rafts. J Biol Chem 277, 39368-39378.

16. Hardy G, Boizel R, Bessard J, Cracowski JL, Bessard G, Halimi S \& Stanke-Labesque F (2005) Urinary leukotriene $\mathrm{E}_{4}$ excretion is increased in type 1 diabetic patients: a quantification by liquid chromatography-tandem mass spectrometry. Prostaglandins Other Lipid Mediat 78, 291-299.

17. Visioli F, Rise P, Barassi MC, Marangoni F \& Galli C (2003) Dietary intake of fish vs. formulations leads to higher plasma concentrations of n-3 fatty acids. Lipids 38, 415-418.

18. Marangoni F, Angeli MT, Colli S, Eligini S, Tremoli E, Sirtori CR \& Galli C (1993) Changes of n-3 and n-6 fatty acids in plasma and circulating cells of normal subjects, after prolonged administration of 20:5 (EPA) and 22:6 (DHA) ethyl esters and prolonged washout. Biochim Biophys Acta 1210, 55-62.

19. Brossard N, Croset M, Pachiaudi C, Riou JP, Tayot JL \& Lagarde M (1996) Retroconversion and metabolism of $\left[{ }^{13} \mathrm{C}\right] 22: 6 \mathrm{n}-3$ in humans and rats after intake of a single dose of $\left[{ }^{13} \mathrm{C}\right] 22$ :6n-3-triacylglycerols. Am J Clin Nutr 64, $577-586$. 